\title{
Bilateral retinoblastoma: the prognosis for vision
}

\author{
CLIVE MIGDAL
}

From the Department of Ophthalmology, St Bartholomew's Hospital, West Smithfield, London EC1A 7BE

SUMMARY In the treatment of retinoblastoma careful case selection is essential. Each eye is assessed individually; the worse affected eye is no longer routinely enucleated in bilateral cases. One hundred and sixteen patients were reviewed to assess the overall visual prognosis. 50\% achieved a final acuity of $6 / 12$ or better in one eye at the age of 8 years, and 2 patients had acuities of $6 / 12$ or better in both eyes. Various methods of treatment are discussed.

In the early 19th century, when retinoblastoma was first recognised, ${ }^{1}$ early enucleation was recommended in an attempt to achieve a cure, because in the preophthalmoscopic era the tumours had usually reached a large size before they were diagnosed.

Diagnostic and treatment facilities have considerably improved over the years, as has the prognosis both for life and vision. The current trend is towards a more conservative approach, with referral to specific centres that are equipped to deal with these tumours. By careful case selection conservative treatment has achieved excellent results without any adverse effect on the survival of the patients. ${ }^{2-5}$ Apart from survival the maximum possible vision after treatment is specially important for children in their upbringing and education. The final vision will be affected by many factors, including the size and site of the tumours, the type of treatment used, the complications of the treatment itself, and the delay in diagnosis. Reese and Ellsworth ${ }^{6}$ classified retinoblastoma for prognosis, assigning the tumours to 5 groups at the time of diagnosis according to size and site.

The purpose of this paper is to review 116 patients treated for bilateral retinoblastoma and to assess their overall visual prognosis and the factors affecting it. The approach to conservative therapy will be discussed.

\section{Material and methods}

The records of 116 patients with bilateral retinoblastoma born between 1960 and 1974 were reviewed. This allowed a minimum age for assessment of 8

Correspondence to Mr C. Migdal, FRCS, 80 Grafton Way, London W1P 5LP. years. Because of the nature of the referral system in this country, particularly in the earlier years, the first eye had often been enucleated by the referring surgeon before he referred the case to a specialist centre. The patients were divided into familial and sporadic cases. The age at the initial visit was noted and number, size, and site of the tumours in each eye recorded.

The treatment policy evolved in this unit ${ }^{7}$ consists of a careful evaluation of each eye under general anaesthesia, followed by the selection of the relevant therapeutic technique, or combination of such techniques-namely, 'whole eye' irradiation with the cobalt beam unit, focal irradiation with cobalt plaques, light coagulation, cryosurgery, enucleation, and cytotoxic drugs. This approach requires the full co-operation of the ophthalmologist, radiotherapist, oncologist, and pathologist. The success or otherwise of the treatment methods was assessed, and the complications encountered were noted.

Visual acuity, charted at the age of 8 years by the standard Snellen's chart and the patient's own spectacles and/or pinhole aperture, provided a measure of the final visual outcome after treatment.

Table 1 Grading system for final acuity

\begin{tabular}{ll}
\hline Visual acuity & $\begin{array}{l}\text { Prognostic } \\
\text { score }\end{array}$ \\
\hline Enucleation & 1 \\
No light perception & 2 \\
Hand movements & 3 \\
Counting fingers & 4 \\
$6 / 60-6 / 36$ & 5 \\
$6 / 24-6 / 18$ & 6 \\
$6 / 12-6 / 9$ & 7 \\
$6 / 6$ & 8 \\
\hline
\end{tabular}




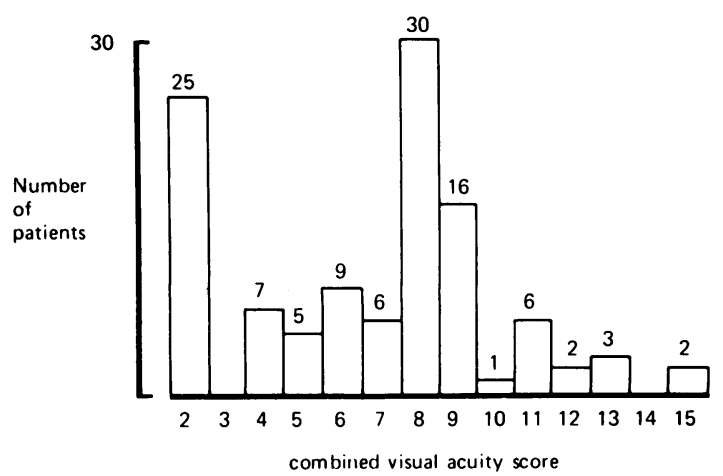

Fig. 1 Combined visual acuity score.

To assess the overall visual prognosis for all the patients a grading system was devised whereby a score was allocated to the final acuity (Table 1). The score for the 2 eyes of each patient was then added, the total giving a figure for the combined acuity of the 2 eyes. The lower the score the worse the combined acuity. For example, a score of 2 means both eyes enucleated while a score of 15 means an acuity of $6 / 6$ in one eye and at least 6/12 in the second (Fig. 1).

\section{Results}

Of the 116 patients with bilateral retinoblastoma 19 (25\%) had a positive family history of the disease, while $87(75 \%)$ were sporadic cases. An equal number (4 each) of familial and sporadic cases initially presented with unilateral retinoblastoma but later developed tumours in the second eye. This emphasised the necessity for repeated careful observation of the 2 eyes in any case with unilateral or bilateral retinoblastoma.

\section{SITE OF TUMOUR}

The final visual acuity is related to the site of the tumour in Table 2 . Of 100 eyes initially treated by conservative methods (though 25 of these later required enucleation) 19 contained tumours situated at the disc or posterior pole, 45 had tumours between the posterior pole and equator, 11 had tumours anterior to the equator, and 25 were situated in a combination of the above. It is noteworthy that no eyes with tumours confined to the area anterior to the equator required enucleation. As would be expected, those eyes with anterior tumours retained good visual acuity on the whole (the majority have a Snellen's acuity of 6/12 or better) but in addition a significant number ( 25 eyes) with tumours between the posterior pole and equator and even some (5 patients) with tumours at the disc or posterior pole had acuities of $6 / 12$ or better after treatment.

\section{TREATMENT}

At the age of 8 years 15 patients retained both eyes, 76 had had one eye enucleated, and 25 patients had undergone bilateral enucleations.

Every effort was made to treat the neoplasms conservatively if possible, provided no risk to life was involved. Forty-four eyes required local treatment with light coagulation alone, cryotherapy alone, or a combination of the two, while 45 eyes were treated with cobalt plaques alone. Those eyes not suitable for focal treatment required external beam radiotherapy.

Twenty-four eyes underwent conservative therapy initially but later required enucleation. In assessing the total number of eyes enucleated it must be noted that a large number of patients had already had one eye enucleated by the referring surgeon. It is possible that some of the eyes could have been saved if treated by specialist methods.

\section{COMPLICATIONS OF TREATMENT}

Complications relevant to the final visual outcome occurred in patients undergoing both whole-eye irradiation with the cobalt beam unit and focal irradiation with cobalt plaques. The majority of patients receiving whole-eye irradiation via the anterior field acquired some degree of radiation lens opacities, but not all of these progressed sufficiently to warrant surgical removal. In those who did require surgery the visual outcome was satisfactory on the whole, aphakic correction being achieved with bifocal spectacles. Amblyopia may be a problem in a patient retaining both eyes who develops a unilateral cataract.

Table 2 Site of tumour related to visual acuity

\begin{tabular}{lccccc}
\hline Site of tumour & \multicolumn{2}{l}{ Visual acuity aged 8 years } & & Total \\
\cline { 2 - 5 } & Enucleation & $P L-C F$ & $6 / 60-6 / 18$ & $6 / 12-6 / 6$ \\
\hline Disc to posterior pole & 8 & 2 & 4 & 5 \\
Posterior to equator & 6 & 6 & 8 & 19 \\
Anterior to equator & 0 & 0 & 1 & 10 \\
Combination of $1,2,3$ & 11 & 2 & 5 & 7 \\
Total & 25 & 10 & 18 & 45 \\
\hline
\end{tabular}


Table 3 Visual acuity at 8 years

\begin{tabular}{|c|c|c|}
\hline Visual acuity & Total number of eyes & $\%$ \\
\hline $\begin{array}{l}\text { Enucleation } \\
\text { PL } \\
\text { HM-CF } \\
6 / 60-6 / 36 \\
6 / 24-6 / 18 \\
6 / 12-6 / 9 \\
6 / 6\end{array}$ & $\begin{array}{r}128 \\
9 \\
9 \\
13 \\
13 \\
39 \\
21\end{array}$ & $\begin{array}{r}55.2 \\
3.9 \\
3.9 \\
5.6 \\
5.6 \\
16.8 \\
9.0\end{array}$ \\
\hline
\end{tabular}

Corneal changes in the form of drying of the tear film, vascularisation, keratinisation, and opacification occurred in the patients receiving anterior cobalt beam radiotherapy. The most marked changes appeared to be in the region of the palpebral aperture. Partial lid shrinkage with resultant poor eyelid closure may be relevant in this regard. Photophobia and diminished acuity were the commonest symptoms.

Cobalt plaques larger than $10 \mathrm{~mm}$ in diameter placed anywhere on the sclera will result in vascular complications often several years after otherwise successful therapy, while the smaller plaques, if placed too close to the optic disc or macula, will have a similar effect due to occlusion of the posterior ciliary vessels. Vitreous haemorrhage secondary to vascular changes was an infrequent complication.

OVERALL VISUAL PROGNOSIS

An analysis of the visual acuity in the 332 eyes at the age of 8 years is given in Table $3.25 .8 \%$ of eyes achieved on acuity of $6 / 12$ or better after treatment, though $55.2 \%$ of the total number of eyes required enucleation (24 eyes underwent conservative treatment initially but later required enucleation).

Fifty-eight patients (50\%) achieved a final acuity of $6 / 12$ or better in one eye at the age of eight years, while 2 patients $(1.72 \%)$ had visual acuities of $6 / 12$ or better in both eyes.

The overall visual prognosis by the grading system devised for this study is shown in Fig. 1 .

\section{Discussion}

The prime aim of treatment of retinoblastoma is to preserve life but in addition every attempt is also made to preserve the eye itself and some degree of useful vision. Whether the retinoblastoma is unilateral or bilateral, an approach has evolved in which each eye is assessed on its individual merits when selecting the relevant treatment method. No longer is the one eye in a bilateral case enucleated merely because it is the worse affected, nor the affected eye in a unilateral case, for a unilateral case may later become bilateral, or the so-called 'good' eye of a bilateral case may develop new tumours at the macula or optic disc.

Early and accurate diagnosis is essential, and this requires an examination under full general anaesthesia with the indirect ophthalmoscope and scleral indentation. Only by this method can small or peripheral tumours be detected early so that they are within the range of treatment by relatively noninvasive methods. The importance of a comprehensive fundal examination is emphasised by Ellsworth ${ }^{8}$ who noted that $75 \%$ of patients had tumours situated anterior to the equator.

Infants with a family history of retinoblastoma are examined from the age of 6 weeks, and then at increasing intervals which are modified accordingly if the patient develops a new tumour which requires treatment. The disease in bilateral cases is often asymmetrical, one eye often having smaller tumours when first seen, while a previously unaffected eye may later develop new tumours. Genetic counselling of the parents and the patients themselves, when old enough, is offered.

The selection of a single therapeutic method or a combination of various methods is aimed at dealing with the tumour while minimising the complications. Conservative methods in regular use include 'wholeeye' irradiation, ${ }^{89}$ focal irradiation with a cobalt plaque ${ }^{10}$ light coagulation, ${ }^{11}$ and cryotherapy. ${ }^{1213}$ The relative indications for the various treatment methods have been suggested by Bedford ${ }^{7}$ on the basis of single or multiple tumours, which are graded according to size.

Treatment is time-consuming, and it is therefore important for the family to understand fully what is entailed. At least $50 \%$ of patients in this series achieved an acuity of $6 / 12$ or better in at least one eye, so that the child could lead a normal life and attend a normal school. Two patients in fact had visions of $6 / 12$ or better in both eyes.

Because the above forms of therapy are so successful, with a $96 \%$ 4-year survival in some units, ${ }^{2}$ there has been a move away from the automatic excision of either the affected eye in a unilateral case or the worse eye in a bilateral case. In the series of Bedford et al. ${ }^{5}$ the only indication for enucleation was involvement of the optic nerve.

Certain complications of the therapeutic methods described above may in themselves adversely affect the visual prognosis, even if the tumour has been satisfactorily dealt with. These include anterior segment damage resulting in corneal or lens opacities, seen after anterior field cobalt beam radiotherapy, or posterior segment vascular changes seen after the application of certain cobalt plaques. A full understanding of the effects and side effects of treatment is needed by ophthalmologist, physicist, and radio- 
therapist if complications are to be minimised. The indications used in this unit in deciding what form the treatment should take are aimed at reducing the risk of complications while at the same time preserving vision and curing the tumour.

\section{CONCLUSION}

With modern therapeutic facilities useful vision may be saved in eyes with retinoblastoma, including those with large tumours which might previously have warranted enucleation. The individual assessment of each eye and the careful selection of the therapeutic method applicable enables the maximal vision to be achieved.

The treatment of the cases in this series was carried out by $\mathrm{Mr} \mathrm{H}$. B. Stallard and Mr M. A. Bedford. Without their expertise such satisfactory results would not have been possible.

I am indebted to Mr Bedford for permission to publish these data, and to Miss Sarah Dew for secretarial assistance.

Mr Walter Gregory, research fellow attached to the Research Centre for Mathematical Modelling of Clinical Trials, helped with the statistics.

\section{References}

1 Wardrop J. Observations on the fungus haematodes. Edinburgh: Constable, 1809.
2 Lennox EL, Draper GJ. Sanders BM. Retinoblastoma: a study of natural history and prognosis: 268 cases. $B r$ Med J 1975: 3: 731-4.

3 Abramson DH, Adams K, Ellsworth RM, et al. Simultaneous bilateral radiation for advanced bilateral retinoblastoma. Arch Ophthalmol 1981; 99: 1763-6.

4 Abramson DH, Javitt J, Ellsworth RM, et al. Treatment of bilateral groups I through III retinoblastoma with bilateral radiation. Arch Ophthalmol 1981;99: 1761-2.

5 Bedford MA, Bedotto C, MacFaul PA. Retinoblastoma-a study of 139 cases. Br J Ophthalmol 1971; 55: 19-27.

6 Reese AB, Ellsworth RM. The evaluation and current concept of retinoblastoma therapy. Trans Am Acad Ophthalmol Otolaryngol 1963; 67: 164-72.

7 Bedford MA. The management of ocular malignant disease. In: Perkins ES, Hill DW, eds. Scientific foundation of ophthalmology; London: Heinemann; 1977: 319-23.

8 Ellsworth RM. The practical management of retinoblastoma. Trans Am Ophthalmol Soc 1970; 67: 462-534.

9 Skeggs DBL, Williams IG. The treatment of advanced retinoblastoma by means of external irradiation combined with chemotherapy. Clin Radiol 1966; 17: 169-72.

10 Stallard HBS. The treatment of retinoblastoma. Ophthalmologica 1966; 151: 214-30.

11 Höpping W, Meyer-Schwickerath RG. Light coagulation treatment in retinoblastoma. In: Boniuk $\mathrm{M}$, ed. Ocular and adnexal tumours. London: Kimpton, 1964.

12 Lincoff $\mathrm{H}$, McLean JM, Long R. The cryosurgical treatment of intraocular tumours. Am J Ophthalmol 1967; 63: 389-99.

13 Abramson DH, Ellsworth RH, Rozakis GW. Cryotherapy for retinoblastoma. Arch Ophthalmol 1982; 100: 1253-6. 